\title{
Negative Josephson coupling in the Kondo strong coupling limit
}

\author{
Oron Zachar \\ Laboratoire de Physique des Solides, U. Paris-Sud, 91405 Orsay, France.
}

(15-Oct-98)

\begin{abstract}
We Show that pair hopping through a Kondo singlet give rise to a negative Josephson coupling. Thus, our calculation supports the existance of staggered pair correlations in the strong coupling limit of a one dimensional Kondo lattice.
\end{abstract}

We use a simple strong coupling limit model, of an isotropic single channel Kondo impurity, by which we show that BCS singlet-pair tunneling through a Kondo singlet state results with a negative Josephson coupling between BCS superconducting grains. For a lattice of such impurities (see figure-1), our result implies a superconducting state with gap nodes due to the modulation, or staggering, of the singlet pair correlation function. To avoid possible misinterpretations, we stress that the node in the pair correlation function due to negative Josephson coupling is a node in the pair center-of-mass motion. It should not be confused with a node in the relative pair state (such as p-wave or d-wave), which is usually thought [1] to result from the residual repulsive interactions between electrons on the screened impurity. We remark on the relation of our result to the phase diagram of the 1D Kondo lattice.

In recent years, several theoretical approaches to the Kondo lattice problem have yielded solutions with the interesting property of having a modulation (or staggering) of the pairing order parameter at the Kondo impurities $x_{j}$; That is, the singlet pairing correlation function has the form,

$$
\begin{aligned}
\chi\left(x_{j}-x_{j^{\prime}}\right) & =\left\langle\hat{O}_{i}(x) \hat{O}_{i}^{\dagger}\left(x^{\prime}\right)\right\rangle \\
& =(-1)^{\left(j-j^{\prime}\right)} \chi_{0}\left(x_{j}-x_{j^{\prime}}\right)
\end{aligned}
$$

where $\chi_{0}$ is the usual correlation of the corresponding operator. Coleman et al. [2] have use an unusual mean field theory, where a coherent three-body bound state of the impurity spins with the conduction electrons leads to a state with the superconducting order parameter fully staggered, i.e., the pair hopping amplitude from site to site is negative. Zachar et al. [5] have used a Bosonization approach to a one dimensional Kondo lattice. They found staggered singlet pairing correlations at a special exactly solvable "Toulouse point", which correspond to a large value of the Kondo coupling coefficient. Heid et al. [3] have devised a new Ginzburg-Landau theory in attempt to explain the superconducting transitions in $U P t_{3}, U P d_{2} A l_{3}$ and $U N i_{2} A l_{3}$. The distinctive feature of this theory [3] is a superconducting order parameterwhich resides away from the center of the Brillouin zone. Namely, they have finite center-of-mass momentum or staggered pairing.

In all of the above mentioned theoretical approaches, the staggered correlation were discussed in conjunction with a certain two-channel Kondo [7] feature leading to odd- $w$ (or odd-in- $\mathcal{T}$ ) pairing [7].6]. For an odd- $w$ pair wave function it may be shownthat, the pair transfer energy between two odd- $w$ slabs, i.e., Josephson coupling, is negative-alternatively. Yet, the reverse is not necessarily true; having a staggered singlet pairing does not entail odd- $w$ pairing.

An undercurrent in all of the above approaches is a dominant Kondo singlet resonance, and therefore, should intuitively be supported by a simple strong coupling approximation. Indeed, Coleman et al. [8] have focussed on strong coupling models in which the bare single channel Kondo coupling is turned into an effective multi-channel coupling due to strong repulsive interactions within the conduction electron gas.

In this paper, we consider a simple strong coupling "quantum chemistry" type of model of a single channel Kondo impurity. The ground state on the Kondo impurity is modeled as a single particle singlet boundstate, of one conduction electron with the local impurity spin. This approach can be considered as perturbations about the infinite Kondo coupling limit $\left(J_{K} / t \rightarrow \infty\right)$. We investigate the problem of Josephson coupling between two BCS superconducting grains through an intermediate Kondo impurity site in a Kondo singlet state.

Our main result is that the Josephson coupling is negative across the Kondo impurity, even though there is no odd- $w$ pairing. Therefore, we demonstrate that staggered pairing correlations can be a consequence of the Kondo singlet state and not only of odd- $w$ pairing.

a)
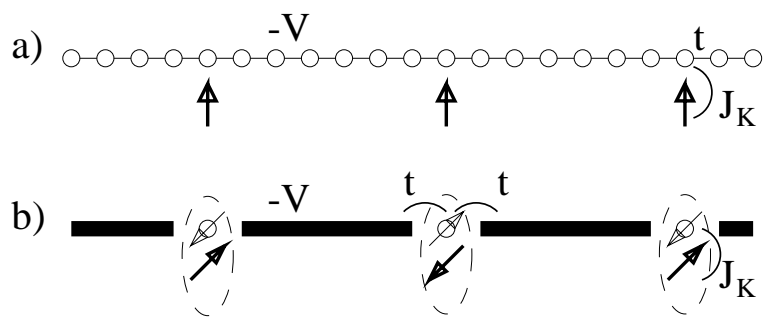

FIG. 1.

Consider a relatively dilute version of the one dimensional Kondo lattice model, where the impurity lattice constant is much larger than the electron lattice constant (see figure-1a). We work in the limit $J_{K} \gg t \gg|-V|$, where $t$ is the hopping matrix element between nearest neighbor electron lattice sites, $J_{K}$ is the Kondo coupling 
between one local moment and one electron site (note: if the Kondo coupling would be to more than one electron site then it will become a multi-channel Kondo model), and $-V<0$ is an attractive interaction between the electrons (and is of conventional origin, whose details are not important for our purpose here). In this strong coupling limit, each Kondo impurity spin bounds a single electron in a singlet state. The rest of the electrons in each segment between impurities are going into a conventional BCS state, and thus can be modeled as a BCS grain (see figure-1b).

The effective Josephson Hamiltonian is

$$
\begin{aligned}
H^{e f f}= & -2 e^{2} \sum_{i j} n_{i}\left[C^{-1}\right]_{i j} n_{j}+\sum_{j} \mu_{j} n_{j} \\
& -\sum_{i j} J_{i j} \cos \left(\theta_{i}-\theta_{j}+\frac{A_{i j}}{\Phi_{0}}\right)
\end{aligned}
$$

where $2 e n_{j}$ is the charge on grain $j, C_{i j}$ is the capacitance, $A_{i j}$ is line integral of a vector potential from grain $i$ to grain $j .\left[n_{j}, e^{i \theta}\right]=\delta_{i j} e^{i \theta}$.

In a Bose liquid, in the absence of a magnetic field, the kinetic energy is unfrustrated in the sense that the ground state is real and nodeless. It is therefore necessary that $J_{i j}>0$. Since $\mathbf{J}_{i j}=\frac{J_{i j}}{\sin \left(\theta_{i}-\theta_{j}\right)}$ is the current across the junction, $J_{i j}$ is a lattice version of the superfluid density $N_{s}(\mathbf{r})$, (i.e. the proportionality constant between the supercurrent and the velocity)

$$
J_{i j} \longleftrightarrow e N_{s}(\mathbf{r})=\frac{\mathbf{J}_{s}(\mathbf{r})}{\mathbf{v}(\mathbf{r})}
$$

Our aim here is to investigate the possibility of having negative Josephson coupling, (or negative superfluid density) $J_{i j}<0$,due to the presence of Kondo impurities in the strong coupling limit.

We investigate the simplest model, in which there are only two superconducting grains. In the usual case, in which direct single-electron tunneling between grains is responsible for Josephson coupling, $J_{12}$ is guaranteed to be positive in the absence of spin-orbit coupling. However, the situation is much richer if we consider the case in which the tunneling is indirect, through a localized state [9]. We consider the effect of a Kondo impurity situated between the two superconducting grains. Above the Kondo temperature, the effect of spin-flip scattering [10] of the tunneling electrons by the impurity spin, (which is equivalent to the effect of spin-orbit coupling), can produce $J_{12}<0$. Typically though, a superconducting transition happens well below the Kondo temperature (e.g., in heavy fermion materials).

To investigate the effect of an intermediate coherent Kondo state on the Josephson coupling, we examine a strong coupling model. In that case, the ground state of the system has a single electron bound in a singlet state with the impurity spin. We supplement the impurity Kondo interaction with a residual repulsive on-site interaction, $U_{i}$, in order to investigate the effect of such a modification, and also in order to keep track of the double occupied impurity states. For simplicity, we neglect direct hopping between the two superconducting grains. In the context of one dimensional systems, such as the Kondo array (see figure), this neglect is justified since the direct Josephson coupling between grains is exponentially small compared with the coupling to the impurity (which is situated in the middle between the left and right "grains").

The impurity spectrum is

\begin{tabular}{|l|l|}
\hline Energy & eigenstates \\
\hline$E_{2}=+\frac{1}{4} J_{K}$ & $|\Uparrow \uparrow \uparrow,| \Downarrow \downarrow\rangle,\left|T_{0}\right\rangle \equiv \frac{1}{\sqrt{2}}(|\Uparrow \downarrow\rangle+|\downarrow \uparrow\rangle)$ \\
\hline$E_{1}=0$ & $|\Uparrow\rangle,|\Downarrow\rangle$ \\
\hline$E_{1}^{\prime}=0+U_{i}$ & $|\Uparrow \uparrow \downarrow\rangle,|\Downarrow \uparrow \downarrow\rangle$ \\
\hline$E_{0}=-\frac{3}{4} J_{K}$ & $\left|S_{i}\right\rangle \equiv \frac{1}{\sqrt{2}}(|\Uparrow \downarrow\rangle-|\Downarrow \uparrow\rangle)$ \\
\hline
\end{tabular}

where $\Uparrow$ is the impurity spin and $\uparrow$ is a conduction electron spin. The Hamiltonian consists of the two superconducting grains, $H_{1}[a]$ and $H_{2}[b]$, the Kondo impurity site interactions $\left(J_{K}\right.$ and $\left.U_{i}\right)$, and hopping interaction, $T$, between the impurity and each grain.

$$
\begin{aligned}
H & =H_{0}+T \\
H_{0} & =H_{1}(a)+H_{2}(b)+\left[J_{K} \tau \cdot \mathbf{S}+U_{i}\left(n_{0}\right)^{2}\right] \\
T & =\sum_{k s}\left[T_{k}\left(b_{k s}^{\dagger} c_{0 s}+c_{0 s}^{\dagger} a_{k s}\right)+\text { h.c. }\right]
\end{aligned}
$$

The lowest order contribution to the Josephson coupling, through intermediate excitations of the impurity state, is forth order in the hopping interaction,

$$
\begin{aligned}
J^{(4)}= & T \frac{1}{w-H_{0}+i \varepsilon} T \frac{1}{w-H_{0}+i \varepsilon} T \frac{1}{w-H_{0}+i \varepsilon} T \\
J \cos ( & \left.\theta_{1}-\theta_{2}\right) \\
= & -\left\langle S_{i}\left|\left\langle\psi_{1}(a) \psi_{2}(b)\left|\hat{J}^{(4)}\right| \psi_{1}(a) \psi_{2}(b)\right\rangle\right| S_{i}\right\rangle \\
= & +\left\langle S_{i}\left|\left\langle\psi_{1} \psi_{2}\left|T \frac{1}{H_{0}} T \frac{1}{H_{0}} T \frac{1}{H_{0}} T\right| \psi_{1} \psi_{2}\right\rangle\right| S_{i}\right\rangle .
\end{aligned}
$$

Where $\left|S_{i}\right\rangle=\frac{1}{\sqrt{2}}(|\Uparrow \downarrow\rangle-|\Downarrow \uparrow\rangle)$ is the impurity singlet ground state, and the $\psi_{j}$ are BCS ground state wave functions on grains 1 and 2 respectively,

$$
\left|\psi_{j}\right\rangle=\prod_{q}\left(u_{q}+e^{i \theta_{j}} v_{q} c_{j q \uparrow}^{\dagger} c_{j-q \downarrow}^{\dagger}\right)|0\rangle .
$$

It is necessary to define a case convention (i.e., the order of spin up/down creation operators). We chose to put up-spin to the left of down-spin operators in the BCS wave-function (8). Thus the proper ordering of the operators that transfer a pair from the left grain to the right grain is $\left\langle\psi_{1} \psi_{2}\left|\left(b_{q \uparrow}^{\dagger} b_{-q \downarrow}^{\dagger} a_{-k \downarrow} a_{k \uparrow}\right)\right| \psi_{1} \psi_{2}\right\rangle$. Direct hoping 
between grains preserves this order, and results with positive Josephson coupling, $J>0$. As we shall see, in tunneling processes through the Kondo impurity site, the ordering can get permuted, leading to a $(-)$ sign. This effect can be traced to the single electron occupancy of the impurity ground state [9].

To fourth order in the hopping interaction, there are 12 different processes that transfer a pair from the left grain to the right grain (and similarly 12 Hermitian conjugate processes that transfer from the right to the left). The 12 processes can be grouped into six pairs of processes with identical amplitudes (one of which has the intermediate impurity spin up, , and the other process has the intermediate impurity spin down, $\Downarrow)$. The contribution of each and every one of the processes is negative, leading to an effective negative Josephson coupling across the Kondo impurity. This is our main result.

$$
\begin{gathered}
J^{e f f}=\left(J_{1}+J_{2}+J_{3}+J_{4}+J_{5}+J_{6}\right) \\
=-\mathcal{M} \sum_{k q}\left|T_{k} T_{q}\right|^{2}\left(v_{q}^{*} u_{k}^{*} v_{k} u_{q}\right) \\
\mathcal{M}=\left[\begin{array}{c}
\quad+\frac{1}{\left(\varepsilon_{k}+\frac{3}{4} J_{K}\right)\left(J_{K}+\varepsilon_{k}+\varepsilon_{q}\right)\left(\frac{3}{4} J_{K}+\varepsilon_{q}\right)} \\
\quad+\frac{1}{\left(\varepsilon_{k}+\frac{3}{4} J_{K}+U_{i}\right)\left(J_{K}+\varepsilon_{k}+\varepsilon_{q}\right)\left(\frac{3}{4} J_{K}+\varepsilon_{q}\right)} \\
+\frac{1}{2} \frac{1}{\varepsilon_{k}+\frac{3}{4} J_{K}+U_{i}}\left(\frac{1}{\varepsilon_{k}+\varepsilon_{q}+J_{K}}+\frac{1}{\varepsilon_{k}+\varepsilon_{q}}\right) \frac{1}{\frac{3}{4} J_{K}+\varepsilon_{q}} \\
+\frac{1}{\left(\varepsilon_{k}+\frac{3}{4} J_{K}\right)\left(J_{K}+\varepsilon_{k}+\varepsilon_{q}\right)\left(\frac{3}{4} J_{K}+U_{i}+\varepsilon_{k}\right)} \\
+\frac{1}{\left(\varepsilon_{q}+\frac{3}{4} J_{K}+U_{i}\right)\left(J_{K}+\varepsilon_{k}+\varepsilon_{q}\right)\left(\frac{3}{4} J_{K}+U_{i}+\varepsilon_{k}\right)} \\
+\frac{1}{2} \frac{1}{\varepsilon_{k}+\frac{3}{4} J_{K}}\left(\frac{1}{\varepsilon_{k}+\varepsilon_{q}+J_{K}}+\frac{1}{\varepsilon_{k}+\varepsilon_{q}}\right) \frac{1}{\frac{3}{4} J_{K}+U_{i}+\varepsilon_{k}}
\end{array}\right]
\end{gathered}
$$

(Note that, by definition, all the denominators must be positive since we are considering virtual excitations relative to the unperturbed ground-state). The first process (see table (1A) in the appendix), is identical to the negative Josephson coupling derivation in reference [9], which inspired our calculation here. That process would dominate in the limit of large repulsion, $U_{i} \rightarrow \infty$, on the impurity site (as indeed was the case considered in reference [9]). In contrast, we consider the limit where $U_{i} \ll J_{K}$ can take any negligible small value. In that case, all the 12 processes have contribution of the same order of magnitude. In the appendix we list the contributing processes, and show in two explicit examples how the negative sign comes about.

In conclusion, for antiferromagnetic Kondo interactions, $J_{K} / t \gg 1$, all the contributions to the Josephson coupling, from intermediate hopping through the impurity states, $J^{e f f}=J_{1}+J_{2}+J_{3}+J_{4}+J_{5}+J_{6}$, are negative. The residual repulsive interactions on the impurity site, $U_{i}$, have only a quantitative effect.

In a continuum limit, our result can be associated with a recent one dimensional field theoretic derivation by Salkola et al. [12]; that a local electronic state of charge$1 e$ spin-0 leads to a $\pi$-kink in the pairing order parameter.
For a periodic one dimensional Kondo lattice of such impurities (figure-1), in the strong coupling limit, the pair correlation function is staggered, due to negative Josephson coupling across impurity sites, $\chi\left(x_{j}-x_{j^{\prime}}\right)=$ $(-1)^{\left(j-j^{\prime}\right)} \chi_{0}\left(x_{j}-x_{j^{\prime}}\right)$. Where $\chi_{0}$ has power law decay (as in a $1 \mathrm{D}$ negative $U$ Hubbard model).

The form of the staggered BCS pairing, written above, is identical to the one which we found previously in a Bosonization approach to the 1D Kondo lattice [5, 11]. That Bosonization calculation was valid in a limited region of parameter space. The fact that we find the same staggered pairing state in a strong coupling limit (in this paper) leads us to propose that a phase of staggered BCS pairing correlations extends over a wide region of parameter space in the phase diagram of the 1D Kondo lattice model. Note that the staggered pairing order, found in this paper, is in the strong coupling limit of a single channel Kondo lattice, independently of $o d d-w$ pairing and two-channel physics.

Acknowledgments: I thank S. Kivelson for innumerable stimulating discussion about the 1D Kondo lattice, and P. Coleman for helpful comments on odd $-w$ pairing. This work was supported by the TMR Fellowship \#ERB4001GT97294.

\section{A. Appendix: Calculation of Josephson coupling terms}

Below, I give a graphical depiction of the 6 processes $(1 A \rightarrow 6 A)$ which transfer a pair from grain $-S_{1}$ to grain $-S_{2}$, with an intermediate impurity spin- $\Downarrow$ state. (In the initial and final ground states, the impurity site is in a singlet bound state; $\left.\left|S_{i}\right\rangle \equiv \frac{1}{\sqrt{2}}(|\Uparrow \downarrow\rangle-|\Downarrow \uparrow\rangle)\right)$. The left column is the intermediate state energy. Since the initial and final states are the same for all the processes, we indicate them only in the table of the first process:

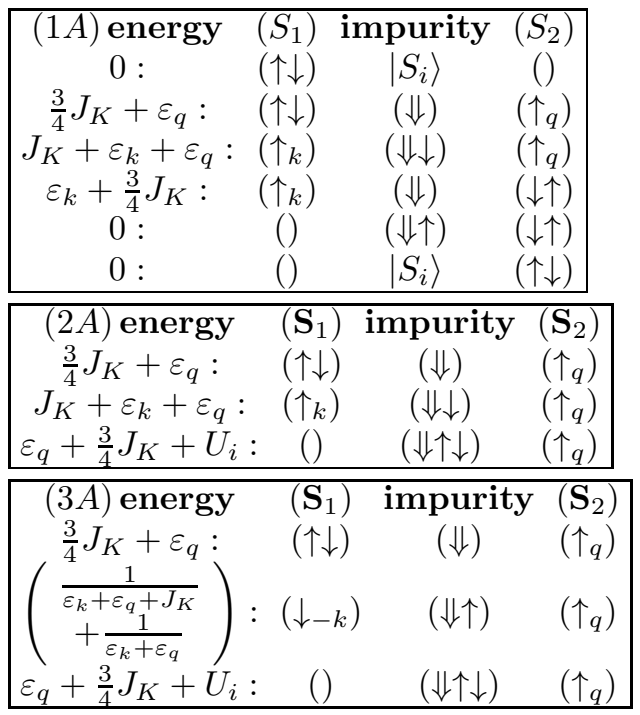




\begin{tabular}{|cccc|}
\hline$(4 A)$ energy & $\left(S_{1}\right)$ & impurity & $\left(S_{2}\right)$ \\
$\frac{3}{4} J_{K}+U_{i}+\varepsilon_{k}:$ & $\left(\uparrow_{k}\right)$ & $(\Downarrow \uparrow \downarrow)$ & () \\
$\varepsilon_{k}+\varepsilon_{q}+J_{K}:$ & $\left(\uparrow_{k}\right)$ & $(\Downarrow \downarrow)$ & $\left(\uparrow_{q}\right)$ \\
$\varepsilon_{k}+\frac{3}{4} J_{K}:$ & $\left(\uparrow_{k}\right)$ & $(\Downarrow)$ & $(\downarrow \uparrow)$ \\
\hline \hline$(5 A)$ energy & $\left(S_{1}\right)$ & impurity & $\left(S_{2}\right)$ \\
$\frac{3}{4} J_{K}+U_{i}+\varepsilon_{k}:$ & $\left(\uparrow_{k}\right)$ & $(\Downarrow \uparrow \downarrow)$ & () \\
$\varepsilon_{k}+\varepsilon_{q}+J_{K}:$ & $\left(\uparrow_{k}\right)$ & $(\Downarrow \downarrow)$ & $\left(\uparrow_{q}\right)$ \\
$\varepsilon_{q}+\frac{3}{4} J_{K}+U_{i}:$ & () & $(\Downarrow \uparrow \downarrow)$ & $\left(\uparrow_{q}\right)$ \\
\hline \hline$(6 A)$ energy & $\left(\mathbf{S}_{1}\right)$ & impurity & $\left(\mathbf{S}_{2}\right)$ \\
$\frac{3}{4} J_{K}+U_{i}+\varepsilon_{k}:$ & $\left(\uparrow_{k}\right)$ & $(\Downarrow \uparrow \downarrow)$ & () \\
$\left(\begin{array}{ccc}\frac{1}{\varepsilon_{k}+\varepsilon_{q}+J_{K}} \\
+\frac{1}{\varepsilon_{k}+\varepsilon_{q}}\end{array}\right):$ & $\left(\uparrow_{k}\right)$ & $(\Downarrow \uparrow)$ & $\left(\downarrow_{-q}\right)$ \\
$\varepsilon_{k}+\frac{3}{4} J_{K}:$ & $\left(\uparrow_{k}\right)$ & $(\Downarrow)$ & $(\uparrow \downarrow)$ \\
\hline
\end{tabular}

I demonstrate the calculation in two cases; In case $1 \mathrm{~A}$, the origin of the (-) sign is from the operator ordering in the BCS wave function of the grains (i.e., to the order of operation $\left.\left\langle\psi_{1} \psi_{2}\left|\left(b_{q \uparrow}^{\dagger} \oint_{-q \downarrow}^{\dagger} a_{-k \downarrow} a_{k \uparrow}\right)\right| \psi_{1} \psi_{2}\right\rangle\right)$. In case $4 \mathrm{~A}$, the $(-)$ sign results from ordering of the electron operators on the impurity site, $\left(1-n_{0 \bar{\sigma}}\right) n_{0 \sigma}=$ $\left(c_{0 \bar{\sigma}} c_{0 \bar{\sigma}}^{\dagger} c_{0 \sigma}^{\dagger} c_{0 \sigma}\right)$. In all of the 12 pair transfer processes, one of these fermion operator orderings takes place, leading to the negative sign.

Case-1A:

$$
\begin{aligned}
& J \cos \left(\theta_{1}-\theta_{2}\right) \\
= & +\left\langle S_{i}\right|\left\langle\psi_{1} \psi_{2}\right| \sum_{k q k^{\prime} q^{\prime}} T_{k^{\prime}}\left(c_{0 \uparrow}^{\dagger} a_{k^{\prime} \uparrow}\right) \times \\
& \frac{T_{q^{\prime}}\left(b_{q^{\prime} \downarrow}^{\dagger} c_{0 \downarrow}\right)}{\left(\varepsilon_{k}+\frac{3}{4} J_{K}\right)} \frac{T_{-k}\left(c_{0 \downarrow}^{\dagger} a_{-k \downarrow}\right)}{\left(J_{K}+\varepsilon_{k}+\varepsilon_{q}\right)} \frac{T_{q}\left(b_{q \uparrow}^{\dagger} c_{0 \uparrow}\right)}{\left(\frac{3}{4} J_{K}+\varepsilon_{q}\right)}\left|\psi_{1} \psi_{2}\right\rangle\left|S_{i}\right\rangle \\
= & +\sum_{k q} \frac{1}{2}\left|T_{k} T_{q}\right|^{2}\left\langle\Downarrow \uparrow\left|\left(c_{0 \downarrow} c_{0 \downarrow}^{\dagger} c_{0 \uparrow}^{\dagger} c_{0 \uparrow}\right)\right| \Downarrow \uparrow\right\rangle \times \\
& \frac{\left\langle\psi_{1} \psi_{2}\left|\left[-\left(b_{q \uparrow}^{\dagger} b_{-q \downarrow}^{\dagger} a_{-k \downarrow} a_{k \uparrow}\right)\right]\right| \psi_{1} \psi_{2}\right\rangle}{\left(\varepsilon_{k}+\frac{3}{4} J_{K}\right)\left(J_{K}+\varepsilon_{k}+\varepsilon_{q}\right)\left(\frac{3}{4} J_{K}+\varepsilon_{q}\right)} \\
= & -\frac{1}{2} \sum_{k q} \frac{\left|T_{k} T_{q}\right|^{2}\left(e^{-i \theta_{2}} v_{q}^{*} u_{k}^{*}\right)\left(e^{i \theta_{1}} v_{k} u_{q}\right)}{\left(\varepsilon_{k}+\frac{3}{4} J_{K}\right)\left(J_{K}+\varepsilon_{k}+\varepsilon_{q}\right)\left(\frac{3}{4} J_{K}+\varepsilon_{q}\right)} .
\end{aligned}
$$

Thus,

$$
J_{1}=-\sum_{k q} \frac{\left|T_{k} T_{q}\right|^{2}\left(v_{q}^{*} u_{k}^{*} v_{k} u_{q}\right)}{\left(\varepsilon_{k}+\frac{3}{4} J_{K}\right)\left(J_{K}+\varepsilon_{k}+\varepsilon_{q}\right)\left(\frac{3}{4} J_{K}+\varepsilon_{q}\right)} .
$$

The factor $\left|T_{k} T_{q}\right|^{2}\left(v_{q}^{*} u_{k}^{*} v_{k} u_{q}\right)$ is common to all the terms. All we need to do is figure out the sign of the permutation to canonical order of operators and the energy denominator of each possible tunneling sequence.

Case-4A:

$$
J=+\sum_{k q} \frac{1}{2}\left|T_{k} T_{q}\right|^{2}\langle\Downarrow \uparrow|\left\langle\psi_{1} \psi_{2}\right|
$$

$$
\begin{aligned}
& \frac{\left(c_{0 \uparrow}^{\dagger} a_{k \uparrow}\right)\left(b_{-q \downarrow}^{\dagger} c_{0 \downarrow}\right)\left(b_{q \uparrow}^{\dagger} c_{0 \uparrow}\right)\left(c_{0 \downarrow}^{\dagger} a_{-k \downarrow}\right)\left|\psi_{1} \psi_{2}\right\rangle|\Downarrow \uparrow\rangle}{\left(\varepsilon_{k}+\frac{3}{4} J_{K}\right)\left(J_{K}+\varepsilon_{k}+\varepsilon_{q}\right)\left(\frac{3}{4} J_{K}+U+\varepsilon_{k}\right)} \\
= & +\sum_{k q} \frac{1}{2}\left|T_{k} T_{q}\right|^{2}\left\langle\Downarrow \uparrow\left|\left[-\left(c_{0 \downarrow} c_{0 \downarrow}^{\dagger} c_{0 \uparrow}^{\dagger} c_{0 \uparrow}\right)\right]\right| \Downarrow \uparrow\right\rangle \\
& \times \frac{\left\langle\psi_{1} \psi_{2}\left|\left(b_{q \uparrow}^{\dagger} b_{-q \downarrow}^{\dagger} a_{-k \downarrow} a_{k \uparrow}\right)\right| \psi_{1} \psi_{2}\right\rangle}{\left(\varepsilon_{k}+\frac{3}{4} J_{K}\right)\left(J_{K}+\varepsilon_{k}+\varepsilon_{q}\right)\left(\frac{3}{4} J_{K}+U+\varepsilon_{k}\right)} .
\end{aligned}
$$

Thus

$$
J_{4}=-\sum_{k q} \frac{\left|T_{k} T_{q}\right|^{2}\left(v_{q}^{*} u_{k}^{*} v_{k} u_{q}\right)}{\left(\varepsilon_{k}+\frac{3}{4} J_{K}\right)\left(J_{K}+\varepsilon_{k}+\varepsilon_{q}\right)\left(\frac{3}{4} J_{K}+U+\varepsilon_{k}\right)} .
$$

[1] C.M. Varma, Physica B 148, 17-21 (1987).

[2] P. Coleman, E. Miranda and A. M. Tsvelik, Phys. Rev. Lett. 70, 2960 (1993); Phys. Rev. B49, 8955 (1994).

[3] R. Heid, Ya. B. Bazaliy, V. Martisovits, and D. L. Cox, Phys. Rev. Lett. 74, 2571 (1995).; V. Martisovits, and D. L. Cox, "Staggered pairing phenomenology for $\mathrm{UPd}_{2} \mathrm{Al}_{3}$ and $U N i_{2} \mathrm{Al}_{3}$ ", cond-mat/97...

[4] Cox DL; Makivic M., PHYSICA B, 1994 APR, V199 APR:391-395.

[5] O. Zachar, S. A. Kivelson and V. J. Emery, Phys. Rev. Lett. 77, 1342 (1996).

[6] V.L. Berezinski, JETP Lett. 20, 287 (1974); E. Abrahams, A. V. Balatsky, Phys. Rev. B 45, 13125, (1992) ; E. Abrahams, A. Balatsky, J. R. Schrieffer and P. B. Allen, Phys. Rev. B47, 513 (1993).

[7] V. J. Emery and S. A. Kivelson, Phys. Rev. B 46, 10812 (1992).

[8] P. Coleman, A.M. Tsvelik, N. Andrei and H.Y. Kee, cond-mat/9707002 ; P. Coleman, A.M. Tsvelik, condmat/9707003.

[9] B.I. Spivak and S.A. Kivelson, Phys. Rev. B, V43 N4, 3740 (1991) ; S.A. Kivelson and B.I. Spivak, Phys. Rev. B, V45 N18, 10490 (1992).

[10] L. Bulaevski, V. Knzii and A. Sobbianin, JETP Lett., V25 N7,314 (1977); L. I. Glazman and K.A. Matveev, JETP Lett., V49, 659, (1989).

[11] Oron Zachar, "Staggered liquid phases of the 1D Kondo lattice", unpublished.

[12] M. Salkola and J.R. Schrieffer, "Topological excitations of one-dimensional correlated electron systems", unpublished. 